\title{
Measurement-Based Effective Conductivity of Carbon Nanotube Networks for Microwave Design Purposes
}

\author{
Emmanuel Decrossas $^{\# 1}$, Mahmoud A. EL Sabbagh* ${ }^{2}$, Victor Fouad Hanna ${ }^{+3}$ and Samir M. El-Ghazaly ${ }^{\sharp 4}$ \\ ${ }^{\#}$ Dept. of Electrical Engineering, University of Arkansas, Fayetteville, AR, 72701, USA \\ 1 edecrossas@ieee. org \\ 4 elghazal@uark. edu \\ ${ }^{*}$ Dept. of Electrical Engineering and Computer Science, Syracuse University, NY, 13244, USA \\ 2 msabbagh@ieee.org \\ Université Pierre et Marie Curie - Paris 6, EA 2385, L2E, F-75252, Paris, France \\ ${ }^{3}$ victor.fouad_hanna@upmc.fr
}

\begin{abstract}
The conductivity of carbon nanotube (CNT) networks is investigated in this work. A mathematical model based on the percolation theory is developed to be implemented in simulation software to design CNT-based high-frequency components. For this purpose, two independent measurement setups are used to extract the conductivity of carbon nanotubes in its dry powder form as furnished by the manufacturer. Moreover, the radio frequency-dependent properties of CNT networks are studied experimentally for different packing densities. The effective conductivity of CNTs is extracted based on the percolation theory which provides a physicalmathematical model.
\end{abstract}

Index Terms - Carbon nanotube networks, conductivity, microwave measurements, percolation theory, permittivity extraction.

\section{INTRODUCTION}

After the discovery of carbon nanotubes (CNTs) in the early nineties [1], researchers explored new fabrication technique to enhance the uniformity, length, and yield. The current, research is more oriented toward rigorous characterization techniques and exploration of new applications [2]. The electrical characterization including the knowledge of the complex permittivity and conductivity at high frequencies is a necessary step to understand and develop novel future carbon nanotube-based components. The objective of this work is to rigorously characterize CNT networks at microwave frequency to improve/develop carbon nanotube based circuits. The implementation of physicalmathematical models based on experimental data improves the accuracy of the simulation of new microwave designs and reduce the time/cost of the device fabrication and provide a better understanding of the electromagnetic-material interaction. Commercial CNT are usually available in a powder form where both semiconducting and conducting CNTs are entangled and randomly distributed. In this work, we used carbon nanotubes in a dry powder form as furnished by the supplier without any processing to characterize the conductivity at radio frequencies (RF). For this purpose, two different setups are used to extract the conductivity of carbon nanotube networks: 1) CNTs are packed in a shorted circular waveguide connected to a performance network analyzer via coaxial cable to extract the effective complex permittivity from the reflection coefficient $\left(S_{11}\right)$ [3]. 2) CNTs replace the metallic trace in planar transmission lines and extraction is based on reflection and transmission measurements [4]. The combination of the data extracted from the CNT powder using two completely different methods is used to provide a general mathematical formula of the effective conductivity function of the packing density and frequency. The variation of the conductivity is explained based on the percolation theory. The percolation control parameters are then found by fitting the measurements curve. The usage of two independent procedures is general enough to minimize any possible fluctuations in results due to random orientation, alignment and/or the number of semiconducting versus conducting nanotubes included within the CNT networks.

\section{DESCRIPTION OF COAXIAL-CIRCULAR WAVEGUIDE DISCONTINUITY MEASUREMENT SETUP}

The first method is a probe measurement where a custom designed circular waveguide is shorted at the end and filled with the carbon nanotube networks to be tested. The circular waveguide is connected to the network analyzer via a male precision adapter. The pin of the male part protruding in the circular waveguide plays the role of the probe. Extraction of the complex effective permittivity is then carried out using the

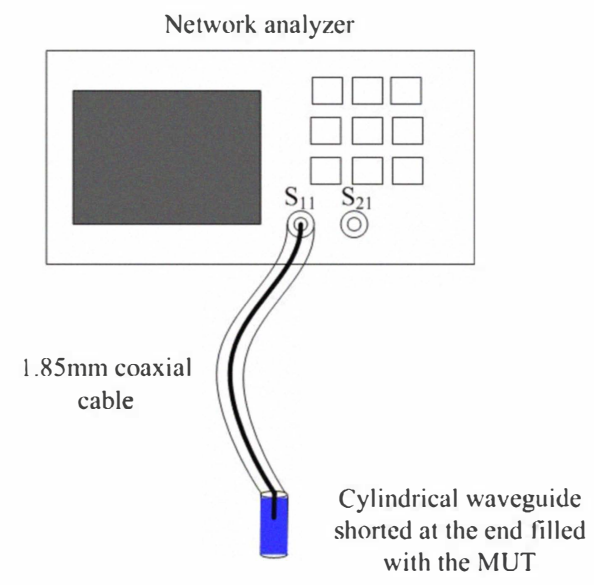

Fig. 1: Schematic of the measurement setup where a cylindrical waveguide filled with material under test is connected to a network analyzer via a $1.85 \mathrm{~mm}$ coaxial cable. 
process described in [3]. The conductivity is computed from the imaginary part of the extracted complex effective permittivity $\left(\varepsilon=\varepsilon^{\prime}-j \varepsilon^{\prime \prime}\right)$ using the following relation:

$$
\sigma=\omega \varepsilon_{0} \varepsilon^{\prime \prime}
$$

where $\omega$ is the angular frequency and $\varepsilon_{0}=8.85 \times 10^{-12}(\mathrm{~F} / \mathrm{m})$ is the vacuum permittivity. This approach is broadband, non destructive and low cost. It allows the measurement of different packing densities where a small amount of material under test (MUT) stands alone in the circular waveguide. Measurements are realized for different packing densities up to $0.4 \mathrm{~g} / \mathrm{cm}^{3}$ where the powder becomes so compact that the pin cannot be easily inserted. Thus, another measurement setup is used to extract the conductivity at higher packing density where the metallic trace of a transmission line is replaced by carbon nanotubes.

\section{DESCRIPTION OF THE TRANSMISSION LINE MEASUREMENT SETUP}

The transmission-line measurement setup consists of a stripline structure where the CNTs replace the metallic trace of the stripline structure shown in Fig. 2. The reflection $\left(S_{11}\right)$ and transmission $\left(S_{21}\right)$ coefficients are then measured to extract the complex effective permittivity of the metallic trace composed of CNT networks as described in [4].

The conductivity is then computed from the extracted complex effective permittivity using (1). Measurements are performed considering different packing densities but are limited to the frequency band from $10 \mathrm{MHz}$ to $400 \mathrm{MHz}$ where only the fundamental mode propagates in the structure. This technique allows measurements of high packing density close to the theoretical value of the CNT density $1.1 \mathrm{~g} / \mathrm{cm}^{3}$.

\section{EXPERIMENTAL RESULTS}

The conductivity function of the frequency extracted for different packing densities is presented in Fig. 3. It is clear that the conductivity increases with the packing density as

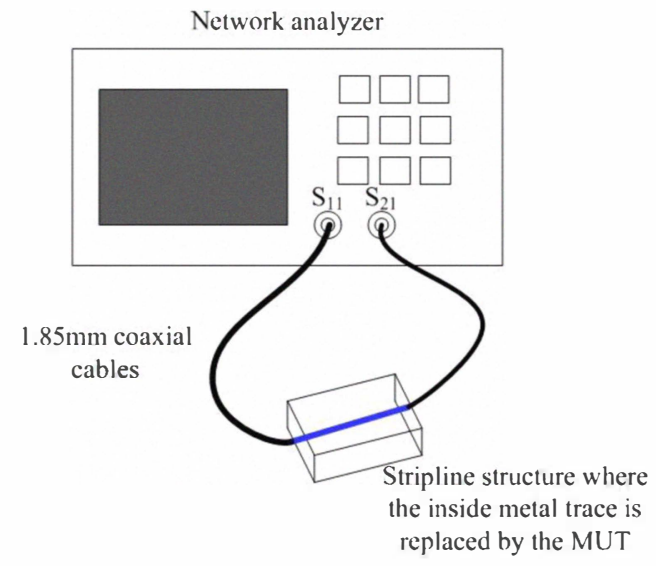

Fig. 2: Schematic of the transmission line measurement where the metallic trace replaced by the MUT inside a stripline structure is connected to the network analyzer via $1.85 \mathrm{~mm}$ coaxial cables. more metallic nanotubes are introduced. In addition, the conductivity decreases with the frequency as the losses due to skin effect and carbon nanotubes semiconducting/conducting interconnects as well as air voids effects become predominant.

The conductivity of carbon nanotubes is extracted over a large range of packing densities at $100 \mathrm{MHz}$ as presented in Fig. 4. From the experimental results, a mathematical model is built from linear regression fitting curves to predict the behavior of the conductivity of the carbon nanotubes. The model is based on the percolation theory [5] that properly predicts the behavior of CNT networks composed of semiconducting/conducing CNTs and air voids. The percolation behavior of the material describes the change of the material from dielectric to conductor due to the insertion of metallic nanotubes which enhances the conductivity of the material.

Above the percolation threshold $p_{c}$, the frequencydependent conductivity of a conductor-insulator mixture can be expressed in terms of a power law relation as:

$$
\sigma_{\mathrm{AC}} \propto\left(p-p_{c}\right)^{t} \text { for } p>p_{c}
$$

where $p$ is the occupation probability and $t$ is the critical exponent. The packing density defined as the ratio of the powder weight to the enclosure volume is adopted in this work as the independent variable describing percolation behavior instead of the volume/weight fraction due to the complex nature of the MUT. The critical exponent $t$ is estimated theoretically to be between 1.6 and 2 for threedimensional percolation networks. However, experiments show that its value varies from 1.3 to 3.1 [6]. For the studied CNTs, the critical exponent obtained by the linear regression fitting curve of the conductivity at $100 \mathrm{MHz}$ shown in Fig. 5 is 2.6 which agrees with the percolation data reported in the literature.

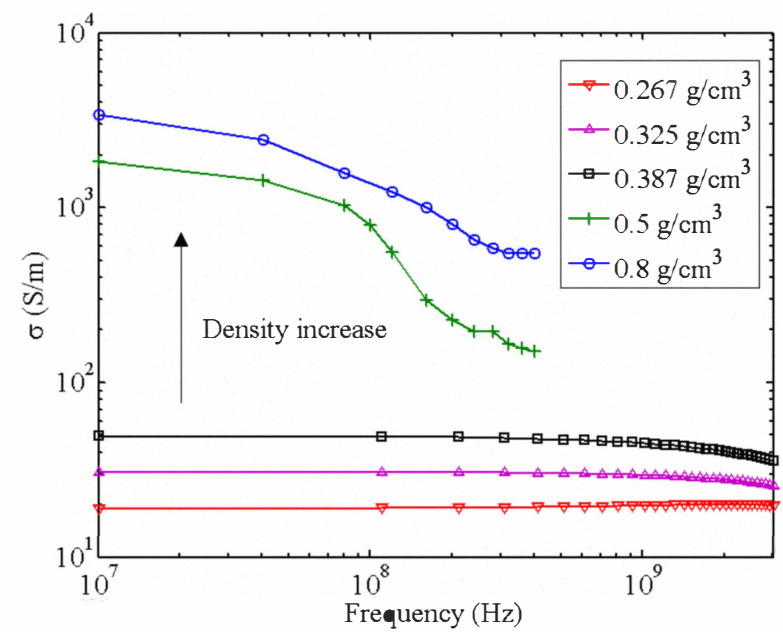

Fig. 3: Extracted conductivity function of the frequency of carbon nanotubes in a dry powder form using the measurement setups presented in Fig. 1 and Fig. 2 for different packing densities. The data obtained for densities below $0.4 \mathrm{~g} / \mathrm{cm}^{3}$ are obtained from the coaxial discontinuity setup while above this density statistical measurements variation are extracted from the transmission line measurement setup. 


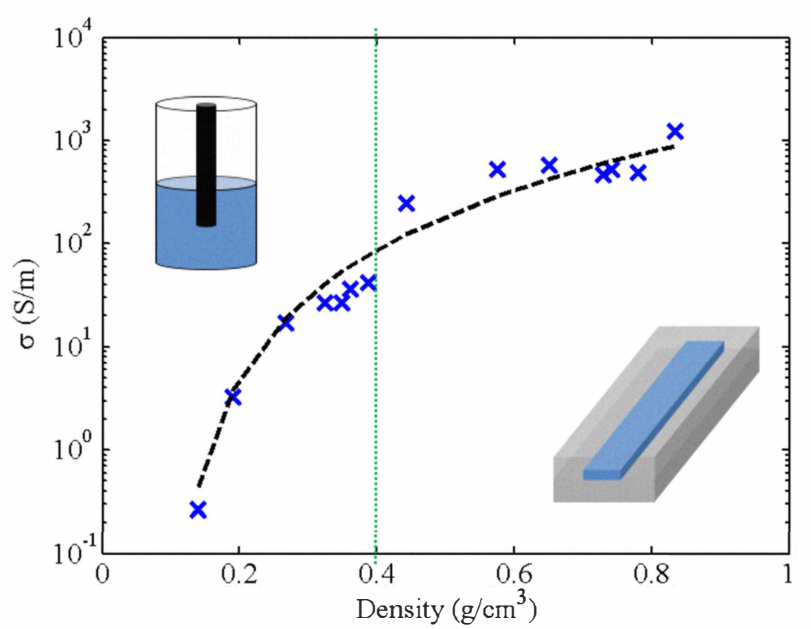

Fig. 4: Semi-log plot of the AC conductivity of CNT networks extracted at $100 \mathrm{MHz}$ using two different extraction measurement setups. The probe measurement is used for packing densities below $0.4 \mathrm{~g} / \mathrm{cm}^{3}$ and the transmission line measurement is used for higher packing densities. The dashed line is obtained from (2) after fitting the extracted conductivity as shown in Fig. 4.

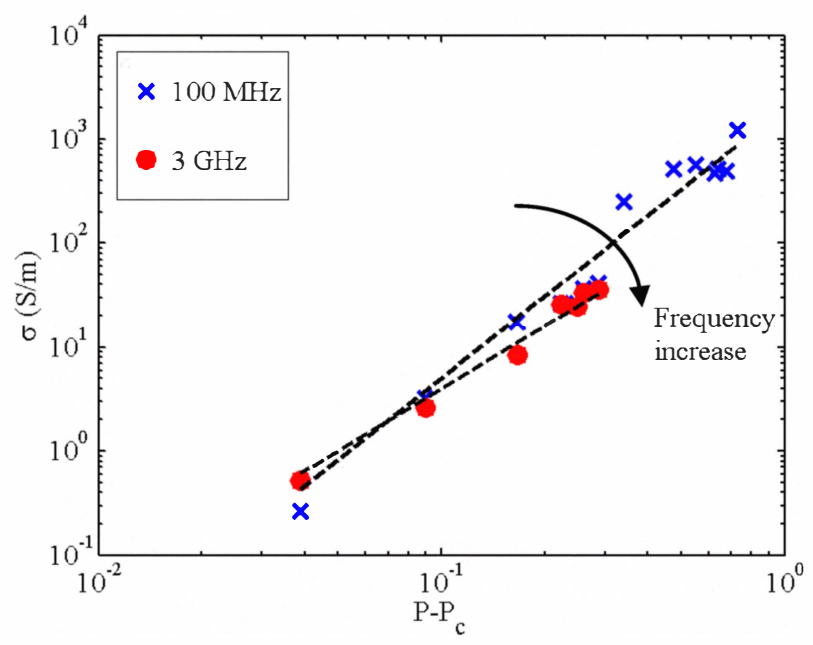

Fig. 5: Log-log plot representing the linear regression fitting curves to find percolation parameters based on the power law described in (2) and data from Fig. 3.

Table I summarizes the percolation parameters using (2) obtained at different frequencies from Fig. 5 and other measurements as well.

TABLE I

SUMMARY OF THE PERCOLATION PARAMETERS OBTAINED FROM LINEAR REGRESSION FITTING CURVES USING (2) AND EXPERIMENTAL RESULTS

\begin{tabular}{ccc}
\hline Frequency $(\mathrm{MHz})$ & Percolation threshold $P_{c}$ & Critical exponent $t$ \\
\hline 50 & 0.1 & 2.6 \\
100 & 0.1 & 2.6 \\
3000 & 0.1 & 2
\end{tabular}

At low frequencies the variation of the conductivity function of the density is higher as the wavelength of the electromagnetic are larger than the separation between two metallic particles. When the frequency range increases to the $\mathrm{GHz}$ range the conductivity decreases as the losses such as skin effect and semiconducting/conducting

CNTs interconnect effects cannot be neglected anymore.

\section{CONCLUSIONS}

The effective conductivity of CNT is modeled based on the percolation theory. Experimental results are obtained using two different extraction methods over a large range of packing densities varying from low values up to the theoretical density of CNTs. The mathematical expression computed from the percolation control parameters after fitting the experimental curves is a real achievement to improve simulation models for future CNTs high-frequency devices. Based on the results presented in this work, it is possible to predict the proper weight of the CNT networks to obtain the desired conductivity. In addition, those results may also lead to new composites material with interesting RF applications by embedding carbon nanotubes in a dielectric host medium. More investigations should be carried out by introducing the high aspect ratio parameters of the CNTs in the percolation formula and try to reduce the errors due to random orientation, alignment and numbers of semiconducting/ conducting carbon nanotubes.

\section{ACKNOWLEDGEMENT}

Research was sponsored by the Army Research Laboratory and was accomplished under Cooperative Agreement Number W91 1NF-10-2-0072. The views and conclusions contained in this document are those of the authors and should not be interpreted as representing the official policies, either expressed or implied, of the Army Research Laboratory or the U.S. Government. The U.S. Government is authorized to reproduce and distribute reprints for Government purposes notwithstanding any copyright notation herein.

\section{REFERENCES}

[1] S. Lijima, "Helical microtubules of graphitic carbon," Nature, vol. 354, pp. 56-58, Nov. 1991.

[2] M. S. Dresselhaus, G. Dresselhaus, and P. Avouris, Carbon Nanotubes Synthesis, Structure, Properties and Applications. Springer-Verlag, Berlin, 2001.

[3] E. Decrossas, M.A. EL Sabbagh, V. Fouad Hanna and S.M. ElGhazaly, "Mode Matching Technique based modeling of coaxial and circular waveguide discontinuities for material characterization purposes," EuMA, International Journal of Microwave and Wireless Technologies, published online 28 September 2011.

[4] M.A. EL Sabbagh and S.M. El-Ghazaly, "Measurement of Dielectric Properties of Carbon Nanotube Networks Used to Build Planar Transmission Lines," in IEEE Int. Symp. Electromagnetic Compatibility, Austin, TX, 17-21 Aug. 2009, pp. 112-117.

[5] D. Stauffer and A. Aharony, Introduction to Percolation Theory, Taylor and Francis, Washington, DC, 1992.

[6] N.K. Shrivastava, B.B. Khatua, "Development of electrical conductivity with minimum possible percolation threshold in multi-wall carbon nanotube/polystyrene composites," in Carbon, vol. 49, no. 13, pp. 4571-4579, 2011. 